Retos, $n^{\circ} \mid 4$, vol.VIII, 2017

\title{
¿Lo volvería a hacer? Revisitando la implementación del paradigma del emprendimiento en una universidad de México
}

\section{Would I do it again? Revisiting the implementation of the entrepreneurship paradigm in a University in Mexico}

\begin{abstract}
Oscar Montiel es líder del Grupo de Investigación Creatividad, Innovación y Emprendimiento y responsable Cap. 20 de Emprendimiento, Incubación y Creación de Empresas de la Academia de Ciencias Administrativas (ACACIA) (México) (oscar.montiel@uacj.mx) (http://orcid.org/0000-0003-0434-1649)
\end{abstract}

Claudia Ivette Rodríguez Lucio es profesora-investigadora de la Universidad Autónoma de Ciudad Juárez (México) (Ivette.rodriguez@uacj.mx) (http://orcid.org/0000-0002-6135-4799)

\begin{abstract}
Resumen
La autoetnografia es un método de investigación reconocido y utilizado en las Ciencias Sociales, aun cuando su uso dentro del área de emprendimiento ha sido escaso y pocos investigadores de esta área han utilizado la propia narrativa (Engstrom, 2012). El presente artículo aborda desde este método las experiencias de dos maestros (Montiel \& Rodríguez, 2016) al realizar la gestión y ser miembros de una academia transversal enfocada al emprendimiento, y narra sus vivencias al impulsarlo como motor del cambio de paradigma de una Universidad pública en México, revisitándolo ahora desde una generalización analítica según lo sugerido por Chang (2008). Los resultados apuntan a realizar una amplia reflexión sobre aspectos organizacionales, sociales y culturales que están inmersos en los procesos de cambio de toda institución, y cómo estos influyen en la intención de modificación del contexto reciente hacia uno donde la creatividad, innovación y emprendimiento sean los ejes que coadyuven a las instituciones en alcanzar y estar a la par de las nuevas políticas nacionales y globales dirigidas hacia la conformación de un ecosistema emprendedor.
\end{abstract}

\begin{abstract}
Autoethnography is a recognized research method used broadly in the social sciences. Its use within the area of entrepreneurship has been scarce, few researchers in this area have used the narrative itself (Engstrom, 2012). This article deals with the experiences of two teachers members of the university's entrepreneurship transversal academy, and its experience in promoting it as the engine of a paradigm shift on a public university in Mexico, revisiting it now using analytical generalization following Chang (2008). The results suggest a broad reflection on organizational, social and cultural aspects, which are immersed in the processes of change in every institution, and how they influence the intention to modify the recent context to one where creativity, innovation and entrepreneurship are the axes that contribute in reach the new national and global policies directed towards the formation of an entrepreneurial ecosystem in a university.
\end{abstract}

\section{Palabras clave | keywords}

Autoetnografía, emprendimiento, transversalidad, universidad emprendedora, ecosistema emprendedor, cambio organizacional.

Autoethnography, entrepreneurship, transversality, entrepreneurial university, entrepreneurship ecosystem, organizational change. 


\section{Introducción}

Oportunidad que no podía pasar. Recién había sido contratado como profesor investigador e iniciaba una vida profesional académica después de laborar 15 años en la empresa familiar. No conocía nada del emprendimiento como constructo, tampoco su medioambiente. Me ofrecían hacerme cargo de la incubadora de empresas de la Universidad. El contexto era muy bueno, había apoyo del gobierno y de la institución. Iniciamos con gran fuerza y empezamos a crecer, tanto dicha área como profesionalmente. Después todo cambio. Se volvió una encrucijada. Por un lado, el gobierno rediseñaba el sistema de apoyo a emprendedores con la constitución del Instituto Nacional del Emprendedor (INADEM). Por el otro, surgió escasez presupuestaria, y apoyar el emprendimiento en la institución era cada vez -y con razón- más difícil. Los organismos acreditadores de la Asociación de Universidades e Instituciones de Educación Superior (ANUIES) empezaban a exigir indicadores de emprendimiento en los programas académicos. Ya en 2011 se había propuesto operar una Academia Transversal de Emprendimiento (ATE), compuesta de maestros de los 4 institutos, y que en dicho seno tuvieran la posibilidad de intercambiar experiencias. Sin embargo, la falta de continuidad en su liderazgo e interés truncó el esfuerzo.

Siendo así, y con algunas personas mirando con escepticismo este proyecto, en septiembre de 2013 soy nombrado -al mismo tiempo que ya lo era de la incubadora- coordinador de la ATE. Sin exagerar, era empezar de cero y en muchas aristas. Ya Sperrer, Müller y Soos (2016) señalan que aún es relativamente escaso el conocimiento sobre mejores prácticas de cómo desarrollar e implementar nuevos programas en emprendimiento en universidades. La presente narrativa es una modesta aportación a ese estado de la cuestión desde una perspectiva no-tradicional: La autoetnografía.

La Universidad tiene cerca de 28000 alumnos. La ATE tenía como misión impulsar y apoyar el desarrollo de un ecosistema y cultura emprendedora en la comunidad universitaria y en la región, y su visión era fomentar una formación integral en los estudiantes, coadyuvando a mejorar la calidad de vida de la región al promover la creación de empresas, intraemprendimiento en las organizaciones e innovación en general, convirtiendo a la universidad en la líder estatal y nacional en la cultura del emprendimiento. Como objetivo a largo plazo, también se 
deseaba cooperar en la construcción de un ecosistema interno de creatividad, innovación y emprendimiento, tomando como base el modelo Rainforest y al Aspen Institute.

Poseía transversalidad académica y multidisciplinariedad (sugeridas por el Banco Mundial y las Naciones Unidas), compuesta por maestros de emprendimiento de los cuatro institutos (Ciencias Administrativas, Ingeniería y Tecnología, Arquitectura Diseño y Arte, y Ciencias Biomédicas). Aproximadamente el $50 \%$ de sus miembros tenía experiencia emprendedora.

Algunas de las actividades surgidas de la ATE fueron:

- Homologación de contenidos de la materia en los 4 institutos, con las últimas metodologías: i) Lean Startup; ii) Opportunity Canvas; iii) Lean Canvas y; iv) Business Model Canvas.

- Conversatorios y actividades formativas del Instituto Mexicano de la Propiedad Industrial (IMPI) a los estudiantes.

- Establecimiento del «Día del Emprendedor», hoy una gestión distinta cambió el nombre, pero el formato, entonces muy criticado, curiosamente es muy semejante. Se realizaron cinco eventos semestrales, 369 proyectos emprendedores, en el que participaron un total de 1292 estudiantes, 3292 asistentes y varias conferencias impartidas.

- Fundación del grupo de investigación Creatividad, Innovación y Emprendimiento.

- Participación exitosa de estudiantes en concursos, obteniendo premios regionales y nacionales.

- Comunicaciones publicadas en congresos e investigaciones difundidas en revistas arbitradas nacionales e internacionales.

- Inicio de la serie de libros «Emprendimiento: Multidimensionalidad, Cambio e Innovación», participando investigadores nacionales e internacionales.

- No se conoce institución nacional o internacional que gestione un modelo como la ATE.

\section{Método}

Autores como Tahar (2009) y Ellis, Adams y Bochner (2010) señalan a la autoetnografía como una estrategia válida de investigación. Aun cuando han existido voces contrarias, la literatura sugiere que es un con- 
texto superado (Denzin y Lincoln, 2000) teniendo un incremento en su uso y aceptación dentro de la investigación antropológica y sociológica (Haluza-DeLay, 2008; Muncey, 2010) y educativa (Starr, 2010).

La autoetnografía implica investigar sobre uno mismo, escribir experiencias que han tenido un impacto significativo en la vida del investigador (Ellis, Adams y Bochner, 2010). Este método fue seleccionado debido a que su proceso da un acceso profundo a dichas vivencias, abordándose simultáneamente el objeto (tema) y el sujeto (investigador), proporcionando un examen de éstas en relación con aquellas similares experimentadas por otros, como medio de encontrar patrones o teorías. Aborda hechos sociales con gran detalle (Pole y Morrison, 2003), son "altamente personalizadas, textos reveladores en los cuales los autores cuentan relatos sobre su propia experiencia vivida, relacionando lo personal con lo cultural" (Richardson, 2003, p. 512), mientras que su fortaleza radica en que comunica "la inmediatez, la fisicalidad y emocionalidad de la experiencia, pero también sus elementos psicológicos y sociales, el diálogo interno del escritor consigo mismo y también situar la experiencia dentro de un amplio contexto social" (AllenCollinson y Hockey, 2005, p.193).

Engstrom (2012) señala que, en años recientes, investigadores en emprendimiento iniciaron un movimiento donde el objetivo es estudiarlo y describirlo bajo estrategias narrativas (Rae y Carswell, 2000; Hjorth y Steyaert, 2004; Berglund, 2007). Por su parte Fletcher (2011) comenta el trabajo seminal de Johannisson, quien investigó el emprendimiento profundizando en su contextualización (comunidades, regiones y familias), abriendo nuevas posibilidades a métodos de investigación que localizaran al investigador como actor participativo.

La autoetnografía reta a la ortodoxia que coloca al investigador como ente neutral y silencioso, lo cual puede dejarlo vulnerable a ser considerado "irracional, particularista, privado y subjetivo, en lugar de ser razonable, universal, público y objetivo" (Greenhalgh, 2001, p. 55). Esto es aún necesario para una variedad de autores que sugieren que las formas de investigar siguen ancladas en presupuestos positivistas y responden a una "ortodoxia metodológica" (Hesse-Biber, 2010, p. 455). El presente artículo -también realizado por Gottlieb, 2015- lo solventa incluyendo un relato de otro miembro de la ATE, lo que provee un ejercicio de reflexión hacia el investigador y el lector. Asimismo, se hace una 
generalización analítica para correlacionar, como sugiere Chang (2008), las vivencias hacia la parte cultural y social, resultando en autoanálisis y transformación (Chase, 2011) y permitiendo una contrastación.

En la autoetnografía puede usarse la primera persona para elaborar el relato (Ellis, Adams y Bochner, 2010) y generalmente ocurre cuando el autor ha observado, vivido o participado de una forma íntima la historia o hecho social que se pretende analizar. Algunas veces se usa la segunda persona para llevar a los lectores hacia la escena, de ir junto con el autor de forma activa, el presenciar la experiencia contada, de ser parte y no estar distante del hecho social relatado (Glave, 2005; McCauley, 1996; Pelias, 2000), incluso utilizan la tercera persona para establecer el contexto, al reportar los resultados y al presentar lo que otros hacen o dicen (Caulley, 2008). En la presente investigación se han seguido dichas recomendaciones, intentando narrar en primera persona la introducción y el relato, mientras que en tercera persona se redactan los apartados de método y conclusiones.

\section{Análisis y resultados}

\subsection{Relato 1}

El cambio de paradigma

Desde Lewin (1951), hasta Cummings y Worley (2007) y Macrì et al. (2002), se ha abordado la resistencia al cambio organizacional, pero una revisión de la literatura sugiere ha sido escasamente abordado en el caso de las universidades.

Bajo sustento científico y después de un proceso de reflexión, pensé que el emprendimiento podía ser una herramienta de cambio para construir capital social, cambiar paradigmas y reconstituir tejidos sociales e industriales a corto, mediano, y largo plazo, tanto para la ciudad como para la propia institución, tomando como referencia a la ciudad de Barcelona, considerada por la Organización para la Cooperación y Desarrollo Económico (OCDE) el modelo a seguir.

Así, me di a la tarea de localizar a los maestros. Descubrí que, en cada instituto, aun cuando los contenidos estaban orientados hacia esa temática, los nombres de dichas asignaturas eran diferentes. En agosto de 2013 se llevó a cabo el primer intento para reactivar la ATE. La 
invitación se realizó por medio de correos electrónicos, sin embargo, la respuesta fue nula. Por tanto, acudí con mi superior a fin de invitar a los maestros por oficio, enviado desde su jefatura inmediata. La respuesta obviamente mejoró: asistieron ocho personas, a quienes en su mayoría no conocía. La buena noticia es que había asistentes de todos los institutos y ello era estratégico para la conformación de la ATE.

¿Cuál era el objetivo? Simple: Insertar a la Universidad en un cambio de paradigma, inquietudes institucionales que empezaban a permear internacionalmente (como por ejemplo lo expuesto por OCDE, 2012). Durante 40 años había desarrollado un mecanismo muy eficiente hacia cubrir las demandas del mercado laboral, pero hoy se veía, como la mayoría de las universidades del país, insertada en una nueva dinámica: generar emprendedores. El contexto exigía indicadores de ello. Simple ¿no? Pero era monumental.

Ya Kuhn (2005) describió los cambios de paradigmas y su dificultad. Tenía que vender la idea de cómo cambiar el nuestro y ello conllevaba una ardua tarea. Era muy consciente que había muchos riesgos. Sacrificar la terminación de mis estudios de doctorado, con el impacto que tendría en mis ingresos -algo nunca valorado por nadie-, tiempo, desarrollar un plan de trabajo incluyente y flexible, y el diseño de estrategias que se implementarían sin experiencia institucional previa.

El primer gran reto era homologar la carta descriptiva, la cual debía reflejar en su contenido lo más reciente en metodologías sobre emprendimiento, enfocado este en un principio a la apertura de empresas. Fue todo un éxito. Los debates eran sumamente enriquecedores. Había puntos de vista y problemáticas particulares de las cuales nadie se percató antes. A diferencia de lo comentado por Nwekeaku (2013), logramos hacer un cambio en el proceso de enseñanza, homologar los contenidos de las materias resultaba relativamente fácil.

Rotación entre institutos

La idea era que los institutos rotaran entre sí la gestión de la ATE a fin de incentivar una dinámica incluyente y transversal. Las tareas se dividieron bajo estos términos, pero no funcionó. Es sistémico. La mayoría de los maestros por honorarios siempre mostraban la mejor disposición, mientras que otros jamás hicieron lo que les fue requerido o su apoyo se reducía a lo indispensable. Por su parte, aquellos que eran de vinculación laboral a tiempo completo, la mayor parte mostraba conductas 
similares, ya que las tareas que realizaban no se reflejaban del todo como actividades valoradas en el programa institucional de estímulos, por lo que no tenían incentivo económico, algo que debería estar concatenado en la estrategia institucional -como lo recomienda la OCDE (2012) -. Las actividades se concentraron en mí y en 5 maestras. Los restantes miembros casi siempre brillaban por su ausencia más no sus críticas, generalmente no constructivas, dentro y fuera de la ATE.

\section{El día del emprendedor}

Un punto angular, ya comentado por la OCDE (2012), era realizar un evento emprendedor incluyente y representativo de nuestra comunidad universitaria, según lo sugerido por Sperrer, Müller y Soos (2016). Lo nombré «Día del Emprendedor» y organizarlo en ese contexto fue muy complicado. No teníamos reconocimiento como ATE en la Universidad, en cada puerta que se tocaba había que explicar sus objetivos y los del evento.

No teníamos recursos o equipo propio (en línea con lo narrado también por Nwekeaku, 2013), todo fue prestado por diversas instancias de la Universidad, quienes muy amablemente y brindándome su confianza, siempre me recibieron con mucha atención y disponibilidad. Salvado este obstáculo, inicié acercamientos ante el empresariado local para que aceptaran asistir como jueces de la competencia. No teníamos claras muchas cosas, así que hice benchmarking con eventos similares realizados en los Estados Unidos. Así nació el «Business Pitch», que realizaban los finalistas de cada categoría.

Se decidió realizar esta actividad semestralmente, a pesar del enorme trabajo y tiempo que suponía. Consideramos en la ATE que mientras no midiéramos que la cultura emprendedora en la Universidad tuviera ya un nivel significativo, era necesario hacerlo así, condición sine qua non para nuestra misión y visión. Los maestros enviaban un listado con los proyectos de sus clases y debían asistir todos.

El número de participantes promediaba 80 proyectos. Se clasificaban en proyectos tradicionales y tecnológicos. Se realizaba en el gimnasio de la Universidad, con capacidad para 5000 personas. El día del evento, abierto al público en general, asistían alrededor de 1500 personas. En ocasiones (segundo y quinto día) hubo apoyo presupuestario para rentar stands para los proyectos. En los otros eventos, hubo que 
solicitar a dos institutos apoyo para que nos prestaran sus mamparas de madera (no en las mejores condiciones y muy pequeñas) para que los estudiantes las usaran como stands. Estéticamente quedaban a deber, pero no había otra forma. Siempre se los agradecimos, la verdad trabajábamos como podíamos.

Los estudiantes se instalaban de 7 a 9 de la mañana. A las 8 yo tenía reunión con los jueces que calificarían la fase A, esto es, a los proyectos a razón de 8-10 stands por juez durante un máximo de 5 minutos en cada uno. Les explicaba qué esperábamos de ellos, cómo calificar, si tenían dudas sobre el formato a utilizar. Para apoyarlos se les asignaban estudiantes voluntarios para acompañarlos en su recorrido. Aquellos con las 15 calificaciones más altas pasaban a la fase B. En ésta realizaban un «Business Pitch» con duración máxima de 2 minutos frente a otro jurado de empresarios. Había tres ganadores (primer, segundo y tercer lugar) en cada categoría.

¿Dificultades? Sí. Enormes. Calaban. Pero podía más la pasión y el compromiso. El primer día se hizo recién 2 meses de haber iniciado la gestión de la ATE, en noviembre del 2013. Fue un parteaguas. Jamás en 40 años la Universidad había hecho algo similar. Lo hicimos en el vestíbulo de su teatro, muy modestamente. Solo eran 29 proyectos provenientes de todos los institutos. Mentiría si digo teníamos claro cómo hacer su gestión, pero era ahora o nunca: teníamos que correr el riesgo; si nos esperábamos, el evento no se realizaría hasta mayo del 2014. Consideramos era demasiado tiempo, teníamos que irrumpir, dar un golpe de timón, mostrar que realmente la ATE bajo mi liderazgo iba en serio y estaba comprometida ante nuestras autoridades.

Para el segundo día nos fuimos a lo grande. Teníamos que seguir innovando, pues somos una gran Universidad con mucho prestigio. Tuve que ir a solicitar fondos de cada instituto para realizar el evento. Alguna gente seguía escéptica que podíamos organizarlo en el espacio más grande e importante de la Universidad. El actual paradigma no veía la dimensión de lo que estaba en juego. Sin embargo, la acogida de los directores de instituto y de la alta gerencia de la Universidad fue sensacional. Todos apoyaron. Todos me dieron su voto de confianza, algo que yo valoraré siempre. Fue desgastante, demasiada responsabilidad. Más de todo, proyectos, empresarios invitados, reconocimientos que imprimir, equipo que pedir prestado, promoción. Todo era 10 veces más. 


\section{El empresariado}

Fue vital, estratégico. Fortaleció las relaciones externas de la institución y el intercambio de conocimiento (OCDE, 2012), y validó muchas dinámicas. Nos dio presencia en toda la ciudad y sobre todo en el sector empresarial, fortaleciendo así nuestra misión y visión institucionales. Rápidamente hice empatía con ellos. Supongo que mi pasado empresarial ayudó. Invitarlos no solo era por estrategia para la construcción del ecosistema interno, sino porque no queríamos que académicos evaluaran los proyectos de emprendimiento, algo que ocurrió durante el primer día. Se prestaba a sesgo y a malos entendidos. Era un intento constante para transparentar procesos.

El invitarlos también era para volver más integral la experiencia de nuestros estudiantes. Que se sintieran exigidos. En la reunión previa les pedí que siempre fueran exigentes al evaluar. No era ni es la única forma de elevar el nivel de los proyectos, lo entendía, sin embargo, era una manera que teníamos al alcance. Siempre tuvieron disposición y la ATE estuvo muy agradecida por ello.

\section{Impacto nacional y binacional}

Lograr que una Universidad norteamericana se interese por nuestras estrategias, hayan viajado para asistir como jueces y pida a partir de ello apoyo para la gestión de su primer «Día del Emprendedor»...no creo sea poca cosa, además sugerido esta internacionalización por la OCDE (2012). También participaron con un capítulo del segundo y tercer libro de la serie, explicados ut supra. Por otra parte, otra institución también norteamericana nos invitó a explorar dinámicas de investigación binacional por medio de dos proyectos, y colaboró con un capítulo en el primer y segundo libro. Algo estábamos haciendo bien.

Institucionalización del día del emprendedor y de la academia Poco a poco, la universidad como institución inició el proceso. La apertura de la jefatura en innovación y emprendimiento fue un paso trascendente. El Instituto de Ingeniería, en lugar de seguir sumándose a la dinámica institucional, inició en paralelo un evento también de corte emprendedor. Varios me decían "es copia del nuestro". ¡A quién le importaba ello! Para mí lo relevante era que iniciará el cambio. En tiempos muy recientes, la ATE fue postulada por la institución para con- 
cursar en el Premio Nacional del Emprendedor del Instituto Nacional del Emprendedor de México (INADEM). Fue el punto más álgido. Sentí que todo el trabajo de la ATE era reconocido.

Hace un año y medio que dejé la coordinación. Ojalá los mecanismos sean los adecuados para que no se pierda el trabajo realizado. Sería una pena, no por mí, sino para la Universidad.

\section{El camino hacia la investigación}

Siempre se invitó a los miembros a realizar investigaciones en el tema. La respuesta fue poca. La gran mayoría de los miembros de la ATE eran maestros de honorarios, por lo que no les generaba retribución -obviamente un emprendedor no lo vería así-. Y en aquellos que eran de tiempo completo, a quienes sí se les reconocía a través del programa de estímulos, tal vez no veían el potencial que podíamos generar. Era empezar de cero, ya que la vía más fácil era pertenecer a algún cuerpo académico consolidado que permitiese el acceso a fondos y redes ya establecidas. Y algunos efectivamente la escogieron.

A pesar de todo ello no desistí y junto con una colaboradora de la ATE nos arriesgamos. Creyó en mí y no podía fracasar. Fue todo un éxito, y de ahí han seguido muchas colaboraciones.

Cuando sentí que la ATE había alcanzado cierta estabilidad y madurez, consideré presentar ante las autoridades de la Universidad la formación de un grupo de investigación en Creatividad, Innovación y Emprendimiento. Por políticas internas, hacía varios años que no se autorizaban. Sin embargo, nuestra propuesta sí lo fue. Ello terminó por impulsar la visión. Ya no era solo una academia de maestros que impulsaban la cultura emprendedora y tenían cierto impacto institucional, sugerido por la OCDE (2012). Iniciábamos una nueva etapa: Generar conocimiento, colocarnos como referentes nacionales e internacionales, bajo una estructura temporal a corto, mediano y largo plazo.

Este grupo fue diseñado siguiendo los principios de la ATE, multidisciplinario. De la serie ya mencionada ya van dos libros editados por la Universidad, construcción de redes nacionales e internacionales, participaciones en congresos, mucho en solo año y medio. En la invitación de presentación del primer libro hubo detalles, pero mi colega y yo, coordinadores de la serie, hicimos caso omiso. No tiene ya importancia. 


\section{La barrera informal}

¿Cómo puede a veces el debate ser de tan bajo nivel? Recuerdo tiempo atrás leí una investigación sobre el mobbing (acoso laboral) en universidades de España ¿Anécdotas? Demasiadas. Desde por qué yo ganaba con mis estudiantes siempre algún premio en los eventos, que había algo raro en ello, que si manipulaba la mesa de evaluación -la cual siempre estuvo con toda intención frente al público, en donde cualquiera podía verificar que las evaluaciones eran capturadas correctamente- ¡hay tantas! Un maestro afirmó incluso que sus alumnos no habían recibido 2 evaluaciones de los jueces solo una, esto a pesar de que la carpeta que contenía éstas estaban frente a él y nunca quiso revisarlas. Todavía comentó que seguramente la segunda había sido hecha a escondidas y en un baño, como si fuéramos ladronzuelos de quinta. Y aquella persona "bueno, pues el representante de Contraloría pues sí estuvo presente, pero no todo el tiempo" ¿Qué culpa tengo de que mis estudiantes iban bien preparados, con ideas innovadoras, siempre intentando trascender, de que fuera exigente con ellos y que los que vociferaban nunca ganaran nada?

Cuando conseguí para el quinto día premios en efectivo para los ganadores sucedió lo mismo: Acusaciones de que se lo iban a gastar en cerveza, que debíamos de supervisar en que lo erogaban...si no había premios que por qué no conseguíamos fondos, y si los conseguimos... no entiendo. ¡Pero ah! En la foto de familia siempre aparecían.

Pero los resultados se iban dando bajo mi liderazgo que, a los 2 años, cuando llegó el momento de relevar la coordinación, la decisión fue reelección con 5 votos contra 3. Por supuesto la impugnaron, aun cuando la autoridad universitaria estuviera presente dando fe, todo bajo reglamento ¿Por qué me reeligieron? Consideraron que aun y cuando se había acordado rotar por instituto la coordinación para sustentar su enfoque transversal -algo que se respetó en los Días del Emprendedor, ya que cada instituto lo organizó- aun había que solidificar algunos elementos del plan, por lo que era mejor no reestructurar hasta que la ATE estuviera más institucionalizada.

¿Por qué el mexicano no tolera el éxito? Ya Paz (1959) habló de ello. Obstáculo cultural y social general comentado por Fogel, Hawk, Morck y Yeung (2005). Junta tras junta, podía palparse el enorme esfuerzo y resultados de nuestro accionar. Supongo es el precio de cambiar un paradigma. No debería ser así. Somos pares, profesionales, 
con un nivel educativo, maestría, doctorado. Si algo, por más pequeño salía mal, intentaban magnificarlo. No vale la pena escribir más de ello. La mediocridad de la que hablaba Ingenieros (2008) ronda siempre. Sabotaje organizacional que describía Young (2000) y Nwekeaku (2013) como resultado de la falta de un cambio en el sistema de valoración informal que existe en toda organización -ya descritos desde su perspectiva en las universidades nigerianas-.

La mayoría de ellos, aquellos que siempre confiaron en mí y me reeligieron, pero sobre todo a mis autoridades que siempre apoyaron, jamás podré pagarles todo el esfuerzo que hicieron y hacen.

Decisión de separación

Todo tiene un final. Era tiempo de separarme de la coordinación de la ATE. Los liderazgos deben terminar en el momento oportuno. Hacia 2 años había cometido el error de no separarme de la coordinación de la incubadora de empresas justo cuando conseguí la acreditación nacional del INADEM. Meses después vinieron los problemas, ajenos a la Universidad que dieron al traste con años de esfuerzo -había construido una red de incubadoras estatal, de las pocas existentes en el país-. Era momento de iniciar un nuevo camino profesional y personal. La autorización del grupo de investigación exigía tiempo completo. El quinto «Día del Emprendedor» había sido todo un éxito. Empresarios, autoridades, estudiantes, en la Academia se sentía el ambiente. En la ceremonia de inauguración era palpable ello. Pero el grupo de investigación necesitaba despegar y desde otra trinchera apoyar la construcción del ecosistema. Había un plan y no podíamos fallar.

Agradecimiento a mi casa: la universidad

Sin duda. La experiencia que mi institución me dio es invaluable. Fue una oportunidad indescriptible. Me hizo tener aún más pasión hacia el emprendimiento y su relevancia empresarial y social. La «camiseta» la tengo tatuada. Suena demasiado político, no me importa. Así es y será.

¿Consideraciones finales? Si algo salía mal, era yo. Si algo salía bien éramos todos ¿Me explico? Pero de nuevo, no había de otra, estaba mi prestigio en juego. No quiero sonar dramático, ni ser un héroe. No es mi intención. Siempre decidí lo último, había mucha responsabilidad hacia la Universidad, hacia las personas que apoyaban, mi vocación 
como investigador, mi ADN emprendedor ¿Qué puedo hacer? Me costó mucho financieramente hablando, roces laborales, momentos incomodos. Pero valió la pena.

Creo que los miembros de una ATE deben ser emprendedores por naturaleza y práctica, de forma que puedan obtener nuevas habilidades. Así también lo sugiere Nwekeaku (2013), pues el emprendimiento resulta vital para el proceso de enseñanza. Un verdadero emprendedor sabe y decide trabajar en equipo, porque es la vía más fácil e inteligente de alcanzar la mayoría de los resultados. Por ello, creo que se debe de elegir $-\mathrm{y}$ no solo por ser docentes de emprendimiento- cuidadosamente a los integrantes de una ATE. Es muy diferente su naturaleza respecto a la mayoría de academias en el nivel universitario.

Dedique casi 3 años. Pero he recibido, aun a pesar de algunas malas experiencias, otras recompensas. Diseñé un seminario virtual de emprendimiento, único en su tipo a nivel internacional, y vienen otros para 2018 y 2019. He sido nombrado para los próximos tres años responsable del capítulo de emprendimiento de la prestigiosa Academia de Ciencias Administrativas A.C. de México (ACACIA). Ser emprendedor está en mis venas. Trascender. Siempre.

¿Lo volvería a hacer? Sí.

\subsection{Relato 2}

Un emprendimiento personal: la ATE

A principios de 2014, siendo docente por honorarios en el programa de Diseño Gráfico y contando con dicha licenciatura y una maestría en investigación relacionada a procesos creativos, por lo general se me asignaban materias teóricas relacionadas con estos temas. Sin embargo, para mi sorpresa e incluso un poco de insatisfacción, al iniciar el semestre se me asignó la asignatura de formación empresarial atribuido, pienso, por contar con una carrera técnica en Administración. Confieso estuve a minutos de pedir que me la retiraran, pues en un compromiso personal me parecía no era la persona indicada. Lo tomé como una oportunidad de salir de mi área de confort y aprender cosas nuevas sin saber que de frente tendría todo un reto que daría un vuelco a mi vida, personal y profesional.

En marzo de 2014 llegué a la ATE por invitación de una docente con quien compartía la impartición de la asignatura. Lo primero que 
me llamó la atención fue la transversalidad de ésta: había maestros de los cuatro institutos, cosa que jamás había vivido en ninguna academia dentro de la Universidad. Era muy interesante escuchar la diversidad de puntos de vista y, aunque en la maestría discutimos sobre transversalidad, interdisciplinariedad y multidisciplinariedad, no fue hasta este momento que tuve la oportunidad de palpar dicho concepto, pues como menciona Bolívar (1998, p. 60) "el discurso de la transversalidad puede quedarse en una práctica discursiva configurado por una retórica política que crea expectativas pero que luego, en la práctica, no puede generar los cambios esperados". Y esto era lo opuesto al objetivo planteado por esta academia y a las acciones que se llevaban a cabo con la finalidad de alcanzarlo, de tal modo que el compromiso, pasión, entusiasmo y sentido emprendedor de los miembros de esta academia, fueron para mí el incentivo más grande para buscar juntos la generación de un cambio de paradigma.

\section{Cambio de paradigma y día del emprendedor}

La Universidad durante años se visualizó bajo un esquema de generadora de empleados para las diferentes necesidades del contexto. Sin embargo, las demandas del mercado actual exigían se redefinieran y reestructuraran las estrategias (OCDE, 2012) que volvieran más competitivos a nuestros alumnos y los ayudaran a adaptarse a los nuevos tiempos. Además, cambios en instancias gubernamentales y educativas exigían una permuta en el esquema actual, pero permear estas transformaciones ha sido sencillo. Girar la dirección hacia la creación de una cultura emprendedora la cual ayudase a gestionar el propio desarrollo e identificar nuevas áreas de oportunidad y ventajas competitivas de ser aprovechadas constituía cuanto menos un reto, porque la mentalidad tenía que modificarse desde el docente. Lo caótico, complejo y falto de linealidad del emprendimiento sugiere que sus maestros deben desarrollar competencias en el descubrimiento, razonamiento e implementación a fin de que los estudiantes puedan sobresalir en los ambientes actuales de gran incertidumbre (Neck y Greene, 2011).

A través del trabajo que se realizaba en la ATE se unificaron contenidos en las cartas descriptivas lo cual permitía que todos los alumnos, aunque las disciplinas fueran distintas, contaran con las mismas habilidades, conocimientos y herramientas para desarrollarse como 
emprendedores. Sin embargo, lograr esto solo dentro del aula no era suficiente. Se tenía que conseguir que el alumno se visualizara y creyera un emprendedor, por lo cual participaban con su proyecto en el evento gestionado por la ATE.

Los retos y dificultades para lograr esto eran grandes. Primero lograr se construyeran buenos proyectos lidiando con deficiencias académicas, en mi caso el área de finanzas. En otros casos el desarrollo visual del proyecto, entre otras cuestiones, que teníamos que solventar con instrucciones rápidas de los temas o con invitaciones de maestros expertos que nos ayudaran en esas áreas que no se dominaban, obstáculos estos ya advertidos por Seikkula-Leino (2007), hasta lograr que el alumno adquiriera y se apasionara con la posibilidad de volver su proyecto realidad. Sin embargo, el trabajo en el aula, aunque arduo, era mucho menos retador que lo que representaba la gestión del evento y todos los obstáculos presupuestarios, de disposición de tiempo y de pasión en el trabajo que se requería para lograrlo. Aunque la ATE estaba constituida por varios miembros, solo pocos manifestábamos un compromiso real y muchas veces sacrificamos familia, tiempo, y dinero por un proyecto que se había planteado en conjunto y por el anhelo de cumplir con aquello trazado.

Evento tras evento se fueron sorteando las dificultades que desgraciadamente no solo provenían de fuera sino algunas de ellas venían del propio seno de la ATE, hasta que llegó el quinto «Día del Emprendedor», evento que obtuvo apoyo del departamento de vinculación de la Universidad, en donde logramos que la estrategia subiera a otro nivel, fuera conocida a través de la institución y entendida como prioridad (OCDE, 2012). En esta actividad se expusieron más de 80 proyectos y se contó con stands para cada uno, donde se sumaron en participación escuelas de media superior, y donde por primera vez se contó con la participación y asistencia de los directores de instituto y el Rector, lo cual indicaba que el trabajo llevado a cabo por fin se visualizaba y reconocía, instalando el evento de forma institucional. Esto claramente reflejaba un cambio de paradigma, un inicio de establecimiento de la cultura emprendedora, nuevas áreas de oportunidad y una educación más integral y de calidad para nuestros estudiantes. El camino fue difícil, definitivamente solo representa el inicio de un transitar que no termina y que debe ajustarse a cambios y condiciones globales actuales. 


\section{Grupo de investigación}

En definitiva, todo lo anterior descrito y vivido, trajo en lo personal la adquisición de conocimiento, habilidades y aptitudes que lograron situarme en posiciones que dieron a mi vida nuevas oportunidades. El área de emprendimiento me apasiona, afortunadamente formo parte del grupo creado a partir de la ATE, donde he participado en diferentes investigaciones, artículos y ponencias, aun cuando para ello se han sorteado diversos obstáculos aun pertenecientes a los viejos paradigmas los cuales no logran comprender la visión holística del emprendimiento y buscan encasillar a un individuo en temas que consideran únicamente pertenecientes a la profesión adquirida (en mi caso diseño, creatividad y publicidad), cuando es precisamente la riqueza de la conjugación de la diversidad de áreas lo que sustenta el crecimiento teórico-práctico de una cultura emprendedora.

Tener un espacio para generar no solo la gestión de esta cultura sino teorizar acerca de la misma, formar parte de redes nacionales e internacionales, la oportunidad de aprender de las personas que estudian el tema, ha sido una oportunidad invaluable. Al día de hoy he coordinado junto a quien fuera coordinador y fundador de esta ATE dos libros de emprendimiento, y otra obra editorial más en proceso de evaluación, la instauración de un seminario virtual de emprendimiento, proyectos en conjunto con la visión de divulgarse y lograr un cambio, y el camino continúa. He descubierto que para trascender hay que arriesgarse, atreverse a ir más allá, desdibujar límites y superar miedos, lo cual no es fácil pero es sumamente enriquecedor, aun cuando esto requiera de un ímpetu que en ocasiones pudiera parecer no fácil de mantener y el cual ha incomodado a terceras personas.

\section{Conclusiones}

En línea con Engstrom (2012), se sugiere debería colocarse en un primer plano la necesidad de penetrar en las experiencias sociales de los emprendedores. Comentan Hjorth y Steyart (2004, p.4) que "Convencer a otros -dirigiendo deseos, organizando recursos, enfrentando obstáculos- y compartiendo imágenes de lo que podría llegar a ser, es realizado en pequeñas narrativas con las cuales la gente puede identificarse". Los eventos y experiencias compartidas en el presente abonaron a la creación de procesos dialécticos (Hargrave y van de Ven, 
2006), y así catalizaron la posibilidad de que emergieran oportunidades e innovaciones institucionales (Ruttan y Hayami, 1984). Al final, el emprendimiento es un hecho social y abordarlo desde diferentes ópticas debería ser una práctica frecuente, académica y pedagógicamente en su enseñanza. Como resultado de revisitar la presente experiencia, y a través de la elaboración de una generalización analítica sugerida por Chang (2008) respecto a realizar autoetnografías analíticas e interpretativas, es menester puntualizar, a) la importancia de manejar mejor las relaciones de poder que se generan hacia el seno y dinámica de grupos o equipos de trabajo, como la ATE y; b) diseñar una estrategia social y organizacional que abordara el profundo cambio institucional que tuvo la Universidad y la cual hubiera facilitado el proceso.

\section{Referencias}

Allen-Collinson, J. \& Hockey, J. (2005). Autoethnography: Self-indulgence or rigorous methodology? En Mike McNamee (Ed.), Philosophy and the sciences of exercise, health and sport: Critical perspectives on research methods (pp.187-202). London: Routledge.

Berglund, H. (2007). Researching entrepreneurship as lived experience. En H. Neergaard y J. P. Ulhoi (Eds.), Handbook of qualitative research methods in entrepreneurship (pp. 75-96). Northampton, MA: Edward Elgar.

Bolívar, A. (1996). Non scholae sed vitae discimus: Límites y problemas de la transversalidad. Revista de Educación, 309, 23-66.

Caulley, D. N. (2008). Making qualitative research reports less boring: The techniques of writing creative nonfiction. Qualitative Inquiry, 14(3), 424-449.

Chase, S. E. (2011). Narrative inquiry: Still a field in the making. En N. Denzin, y V. S. Lincoln (Eds.), The Sage handbook of qualitative research (pp. 421-434). Los Angeles: Sage.

Chang, H. (2008). Autoethnography as Method (Developing Qualitative Inquiry). London: Routledge.

Cummings, T. y Worley, C. (2007). Desarrollo organizacional y cambio. México: Thomson.

Denzin, N. K., y Lincoln, Y. S. (2000). The discipline and practice of qualitative research. en N. K. Denzin y Y. S. Lincoln (Eds.), Handbook of qualitative research (2nd ed., pp. 1-28). Thousand Oaks: Sage.

Ellis, C., Adams, T., y Bochner, A. (2010). Autoethnography: An Overview. Forum: Qualitative Social Research, 12(1). doi: http://dx.doi.org/10.17169/fqs-12.1.1589

Engstrom, C. (2012). An autoethnography account of prosaic entrepreneurship. Tamara Journal for Critical Organization Inquiry, 10(1).

Fletcher D.E. (2011). A curiosity for contexts: Entrepreneurship, enactive research and autoethnography. Entrepreneurship \& Regional Development, 23(1/2), 65-76. 
Fogel, K., Hawk, A., Morck, R. y Yeung, B. (2006). Institutional Obstacles to Entrepreneurship. En Casson, M., Yeung, B., Basu, A. y Wadeson, N. (Eds.), Oxford Handbook of Entrepreneurship. Oxford: Oxford University Press.

Glave, T. (2005). Words to our now: Imagination and dissent. Minneapolis: University of Minneapolis Press.

Gottlieb, F. (2015). Researching entrepreneurship with autoethnography. En Pursuing Innovation Leadership: Proceeding of the 16th International CINet Conference. Continuous Innovation Network (CINet).

Greenhalgh, S. (2001). Under the medical gaze: Facts and fictions of chronic pain. Berkeley: University of California Press.

Haluza-DeLay, R. (2008). Churches engaging the environment: An autoethnography ofobstacles and opportunities. Human Ecology Review, 15(1), 71-81.

Hargrave, T.J., Van de Ven, A.H., 2006. A collective action model of institutionalinnovation. Academy of Management Review, 31, 864-888.

Hjorth, D., y Steyaert, C. (Eds.). (2004). Narrative and discursive approaches in entrepreneurship. Cheltenham: Edward Elgar.

Hesse-Biber, S. (2010). Qualitative Approaches to Mixed MethodsPractice. En Qualitative Inquiry. Thousand Oaks: Sage.

Ingenieros, J. (2008). El hombre mediocre. Ciudad de México: Editorial Porrúa.

Kuhn, T. S. (2005). La estructura de las revoluciones científicas. Madrid: Fondo de Cultura Económica.

Lewin, K. (1951). Field Theory in social science. New York: Harper\&Row.

Macrì D., Tagliaventi M. y Bertolotti F. (2002) Grounded theory for resistance to change in a small organization. Journal of Organizational Change Management, 15(3), 292-310.

McCauley, S. (1996). Let's say. En Patrick Merla (Ed.), Boys like us: Gay writers tell their coming out stories (pp.186-192). New York: Avon.

Montiel, O. y Rodríguez, C. (2016). ¿Lo volvería a hacer? Liderando un cambio de paradigma. El caso de la Academia Transversal de Emprendimiento. En Ojeda, R. y López, L. (Coords.), Gestión Social. Organizaciones Humanas para una Sociedad Incluyente, Memorias del XX Congreso Anual, disponible en http://acacia. org.mx/wp-content/uploads/2016/11/EBook_XX_Congreso_ACACIA.pdf, pp. 7975-8001.

Muncey, T. (2010). Creating autoethnographies. London: Sage.

Neck, H.M. y Greene, P.G. (2011). Entrepreneurship Education: Known Worlds and New Frontiers. Journal of Small Business Management, 49(1), 55-70.

OCDE (2012). A Guiding Framework for Entrepreneurial Universities. Disponible en http://www.oecd.org/site/cfecpr/EC-OECD\%20Entrepreneurial\%20Universities\%20Framework.pdf

$\mathrm{Paz}$, O. (1959). El laberinto de la soledad. México: FCE.

Pelias, R. J. (2000). The critical life. Communication Education, 49(3), 220-228.

Pole, C. y Morrison, M. (2003). Ethnography for education. Maidenhead: Open University Press. 
Rae, D., y Carswell, M. (2000). Using a life-story approach in researching entrepreneurial learning: The development of a conceptual model and its implications in the design of leaning experiences. Education EF Training, 42, 220-227.

Richardson, L. (2003). Writing. A Method of Inquiry. En Denzin, N. y Lincoln, Y. (Eds.), Collecting and Interpreting Qualitative Materials. Thousand Oaks: Sage.

Ruttan, V.W. y Hayami, Y., 1984. Toward a theory of induced institutional innovation. Journal of Development Studies, 20, 203-223.

Schein, E. (1985). Organizational culture and leadership: A dynamic view. Jossey-Bass. USA.

Seikkula-Leino, J. (2007). Opetussuunnitelmauudistus ja yrittäjyyskasvatuksen toteuttaminen (Curriculum reform and entrepreneurship education). Helsinki: Opetusministeriön julkaisuja.

Sperrer, M., Müller, C. y Soos, J. (2016). The Concept of the Entrepreneurial University Applied to Universities of Technology in Austria: Already Reality or a Vision of the Future? Technology Innovation Management Review, 6(10), 37-44.

Starr, L. (2010). The use of autoethnography in educational research: Locating who we are in what we do. Canadian Journal for New Scholars in Education, 3(1), 1-9.

Trahar, S. (2009). Beyond the story Itself: Narrative inquiry and autoethnography in intercultural research in higher education. Forum: Qualitative Social Research, 10(1), Art.30, http://nbn-resolving.de/urn:nbn:de:0114-fqs0901308 [Accesado: 26 de Enero 2016].

Young, A. (2000) I'm just me: A study of managerial resistance. Journal of Organizational Change Management, 13(4), 375-398. 